\title{
Resource-aware Video Multicasting via Access Gateways in Wireless Mesh Networks
}

\author{
Wanqing $\mathrm{Tu}^{1}$, Cormac J. Sreenan ${ }^{2}$, Chun Tung $\mathrm{Chou}^{3}$, Archan Misra ${ }^{4}$, and Sanjay Jha ${ }^{3}$
}

\begin{abstract}
This paper studies video multicasting in large scale areas using wireless mesh networks. The focus is on the use of Internet access gateways that allow a choice of alternative routes to avoid potentially lengthy multi-hop wireless paths with low capacity. A set of heuristic-based algorithms are described that together aim to maximize network capacity: the two-tier integrated architecture algorithm, the weighted gateway uploading algorithm, the link-controlled routing tree algorithm, and the alternative channel assignment algorithm. These algorithms use different approaches to arrange multicast group members into a clustered and two-tier integrated architecture in which network protocols can make use of multiple gateways to improve system throughput. Simulation results are used to determine the performance of the different approaches.
\end{abstract}

\section{INTRODUCTION}

Video streaming represents one of the fastest growing segments of traffic in the Internet today. Multicasting of video over wireless networks poses a difficult set of challenges, both due to the combination of high data rates (relative to wireless capacity) and low latency constraints and the need to support multiple receivers with time-varying link quality. Wireless mesh networks (WMNs) have recently been attracting considerable attention for their ability to offer low-cost connectivity over large urban areas. A WMN consists of a set of mesh nodes, offering connectivity to end user devices (e.g., laptops); the mesh nodes form a relatively-static, multi-hop wireless backbone. Supporting video applications (e.g. online games, large-scale video conference, traffic management, and longdistance learning) in such mesh environments is particularly challenging, given the observed loss in network throughput that occurs when packets traverse multiple successive wireless hops. To support multicast of video in WMN environments, it is thus necessary to explore approaches that can maximize the use of available network resources.

Various papers, e.g. [13-15], have examined the use of multiple wireless transmission rates, multiple radio interfaces, multiple

\footnotetext{
${ }^{1}$ School of Computing and Communication Technology, Glynd $\hat{w} r$ University, Wrexham, United Kingdom. Email: w.tu@glyndwr.ac.uk.

${ }^{2}$ The Mobile \& Internet Systems Laboratory, Department of Computer Science, University College Cork, Cork, Ireland. Email: cjs@cs.ucc.ie.

${ }^{3}$ School Of Computer Science and Engineering, The University of New South Wales, Sydney, Australia. Email: \{ctchou,sanjay@cse.unsw.edu.au\}.

${ }^{4}$ IBM T J Watson Research Center, Hawthorne, New York, USA. Email: archan@us.ibm.com.
}

paths, and the so-called wireless broadcast advantage, to improve wireless network capacity. These approaches are intramesh schemes, in that they aim to support video transmission in WMNs by using wireless resources more effectively. An alternative approach is to enhance WMNs with access gateways that can provide alternative routing paths via the Internet. This can allow a reduction in wireless transmission distance (the number of wireless hops traversed) and hence improve residual capacity. We call this gateway-based approach the integrated architecture and illustrate its potential in Fig. 1. The intra-mesh communication from $S$ to $R$ experiences at least 6 hops, while the integrated paths only take 2 wireless hops (to or from the gateways) because of being able to take advantage of an Internet shortcut via gateways. Besides reduced hop count, an additional set of advantages acrue from the fact that the Internet paths are more stable and usually have greater bandwidth than the wireless links in the mesh network.

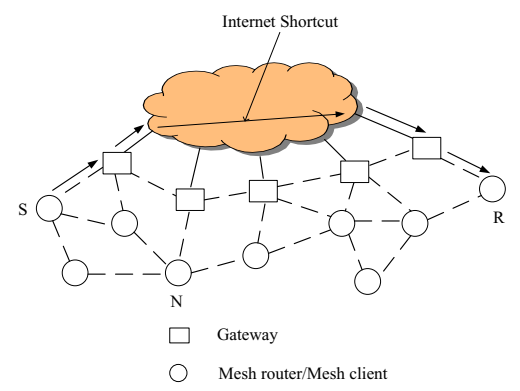

Fig. 1. An example of an Internet shortcut.

Gateway-based approaches [11-12] for routing in WMNs typically address the routing challenge through hierarchical addressing schemes and the use of wired resources in a greedy manner, without consideration of the traffic load and the relative merits of intra-mesh vs. gateway-based paths. In particular, in many instances, the use of an Internet-based path may actually prove counterproductive, especially if the vicinity of the access gateways is congested. In Fig. 1, packets from $S$ prefers the intra-mesh routing to reach $N$ because the intra-mesh routing has the same number of wireless hops as the integrated path but needs no Internet access. Hence, the decison on whether to use the intra-mesh routing or the integrated paths must clearly take into account the sending node, as well as the set of recipients. 
This paper develops a video multicasting framework, for extended WMN environments, that seeks to efficiently exploit available Internet resources while cooperatively sharing the intra-WMN wireless bandwidth. To start, we account for the degradation in video transmisson that arises from its transfer over multiple successive wireless links within the WMN. This observation forms the basis for our resource-aware multigateway WMN video multicasting scheme, which uses a set of inter-linked novel algorithms to construct integrated multicast routes that maximize the network's capacity for QoSsensitive video traffic. The algorithms are based on an WMN architecture where each mesh node has two distinct radios, and include:

- The two-tier integrated architecture (TIA) algorithm establishes a hybrid wired-wireless routing hierarchy. Unlike the schemes in [11-12], TIA employs a threshold of the number of wireless hops to cluster group members into different access areas. The upper tier comprises area gateways, which are used for forwarding traffic between nodes in different lower-tier access areas.

- The weighted gateway uploading (WGU) algorithm is used by a node in an access area to select among the multiple candidate area gateways, so as to avoid routing through "congested" gateways. WGU uses a metric that balances between the wireless distance to the gateway and the gateway's traffic load to ensure high throughput and low latency.

- The link-controlled routing tree (LCRT) algorithm builds a multicasting tree built inside each access area that minimizes the number of forwarding nodes (to reduce the interference-induced delays within the WMN) involved in the dissemination of the multicast traffic.

- The alternative channel assignment (ACA) algorithm exploits the multi-radio architecture of each mesh node by alternatively assigning different sending/receiving channels to mesh nodes, based on their distance (in hops) to the gateway or the multicast sender. This channel assignment strategy promotes more efficient streaming by reducing interference on each successive forwarding hop.

The rest of the paper is organized as follows. Section II assesses related previous work. Section III formulates the problem of degradation of video quality in terms of the number of wireless hops over which it is transported. Section IV then presents our resource-aware multi-gateway WMN video multicasting solution. Computer simulations and evaluation are detailed in Section V. Finally, Section VI concludes the paper.

\section{RELATED WORK}

Research in the area of video multicasting using wireless mesh networks can be classified as being either intra-mesh, where the focus is on the optimisation of wireless links and interfaces, or integrated where the use of Internet gateways is assumed.
Intra-mesh video multicasting utilizes modern wireless techniques such as multiple rate transmission, multiple channels, and wireless broadcast advantage. B. Liu et al [13] proposed the Rate and Contention Aware Multicast (RCAM) scheme that exploits link-rate diversity to construct a multicast forwarding tree, based on the link transmission rates and the associated congestion load expressed via a cumulative transmission time fraction (CTTF) metric. For efficient wireless broadcasting, C. Chou et al [14] suggested the use of link rates that optimize the product of the link rate and the coverage area. Subsquently, Wang et al [15] proposed a fast broadcast tree construction that improves on the rate-coverage scheme. Apart from these papers there has been interesting analytical work regarding the fundamental impact of multiple hops in wireless networks. It was shown in [2] that the per-node multicast throughput of a random multi-hop networks with $n$ nodes, $n_{s}$ multicast sources and $n_{d}$ destinations is bounded by $O\left(\min \left(1, \frac{\sqrt{n}}{n_{s} \sqrt{n_{d} \log n}}\right)\right)$ with a high probability. Therefore the multicast throughput is a decreasing function of the size of the network. This motivates our design goal of limiting the depth of a wireless multi-hop path.

For integrated video multicasting in WMNs, P. M. Ruiz et al [11] proposed a routing mechanism where mesh nodes use prefix continuity-based searches over auto-configured addresses to find their "closest" gateways. Mesh nodes go back to the same gateway in order to connect to Internet form an individual structure with the same address prefix. This work constructs load-independent routing paths. Y. Amir et al [12] presented a hybrid routing protocol for multi-homed wireless mesh networks that provides uninterrupted connectivity and fast handoffs, rather than load-based multicast dissemination.

\section{PROBLEM Formulation}

Within our wireless mesh network suppose a group of $n$ members wish to receive a given video multicast. The members comprise both $G$ gateways and $M$ mesh nodes, so $n=G+M$. We denote the video multicasting group as $V=\left\{g_{0}, g_{1}, \ldots, g_{(G-1)}, m_{0}, m_{1}, \ldots, m_{(M-1)}\right\}$, where $g_{i}(i \in$ $[0, G-1])$ is the $i$ th gateway and $m_{j}(j \in[0, M-1])$ is the $j$ th mesh node. From this point on, we will just use the term "group members" to refer to both WMN gateways and mesh nodes that are members of $V$. In our current work we furthermore assume that each node in the network has two single-channel radio interfaces, allowing simultaneous reception and transmission. Table I lists the symbols used in this paper.

As stated earlier, the key innovation in this work is to exploit wired network resources so as to allow video multicasting in large scale wireless networks without suffering the degradation that results from the use of a large number of successive wireless hops. In general, the wireless video communication cost that causes the degradation of wireless video signals during transmission can be broken down to throughput cost 
TABLE I

SYMBOL LIST

\begin{tabular}{|c|c|}
\hline$V$ & The video session / multicasting group \\
\hline$s$ & The source node of $V$ \\
\hline$n$ & Size of $V$ \\
\hline$G$ & Number of gateways in $V$ \\
\hline$M$ & Number of mesh nodes in $V$ \\
\hline$g_{i}$ & The $i$ th $(i \in G)$ gateway in $V$ \\
\hline$m_{j}$ & The $j$ th $(j \in M)$ mesh node in $V$ \\
\hline$c_{1}$ & Identifier for radio channel on 1 st interface \\
\hline$c_{2}$ & Identifier for radio channel on 2nd interface \\
\hline$K$ & Threshold of the number of wireless hops \\
\hline$k$ & Cumber of wireless hops from a video source to its area gateway \\
\hline$I_{l}$ & Cransmission rate of $V$ \\
\hline$r_{V}$ & Number of wireless links on path \\
\hline$L$ & Number of packets transmitted \\
\hline$P$ & Capacity of link $l$ \\
\hline$C_{l}$ &
\end{tabular}

$\left(\delta_{T}\right)$, delay cost $\left(\delta_{D}\right)$ and delay jitter cost $\left(\delta_{J}\right)$. These three elements can be formulated as

$$
\begin{gathered}
\delta_{T}=\sum_{l=0}^{L-1}\left[\left\{r_{V}-C_{l}\right\}^{+}+I_{l}\right], \\
\delta_{D}=\frac{\delta_{T}}{r_{V}}, \\
\delta_{J}=\sum_{p=1}^{P-1}\left|\left(\delta_{D}\right)_{p}-\left(\delta_{D}\right)_{p-1}\right| .
\end{gathered}
$$

where $L$ is the number of wireless links used to transmit a video session $V, r_{V}$ is the transmission rate of $V, C_{l}$ is the current throughput of the $l$ th link, $I_{l}$ is the capacity loss caused by interference and contention at the $l$ th link, and $P$ is the total number of packets transmitted by $V$. The expression $\left\{r_{V}-\right.$ $\left.C_{l}\right\}^{+}$represents the rate cost of $V$ at the $l$ th link. When $r_{V}>$ $C_{l},\left\{r_{V}-C_{l}\right\}^{+}=r_{V}-C_{l}$; when $r_{V} \leq C_{l},\left\{r_{V}-C_{l}\right\}^{+}=0$. The three equations show that the improvement of $\delta_{T}, \delta_{D}$, and $\delta_{J}$ fundamentally depend on the three factors $L, C_{l}$, and $I_{l}$. According to Equation (1.1), higher video multicasting capacity can reduce the wireless hop count, because the decreased $L$ reduces both the video transmission cost and the associated interference. Previous proposals for shortest path intra-mesh routing can extend WMN range quantitatively by minimising $L$ (choosing longer hops), but suffer from the reduced capacity caused by the use of slower speed links. Hence, our first objective is to use Internet shortcuts to reduce the total number of wireless hops traversed by the video traffic.

The equations show that the large $C_{l}$ at each individual link would reduce the costs of throughput $\left(\delta_{T}\right)$, delay $\left(\delta_{D}\right)$ and delay jitter $\left(\delta_{J}\right)$. As shown in Fig. 2, in integrated wireless communications, gateways connecting to the Internet are likely to become "bottlenecks" because they carry both outbound traffic for remote mesh nodes to the Internet (e.g., $O F_{1}$ and $O F_{2}$ ) and inbound traffic from the Internet (e.g., $I F_{1}$ and $I F_{2}$ ) from the Internet to its child members. Hence, our second objective is to avoid "busy" gateways.

To achieve higher throughput and lower delay and jitter costs, we also require low capacity cost at each wireless

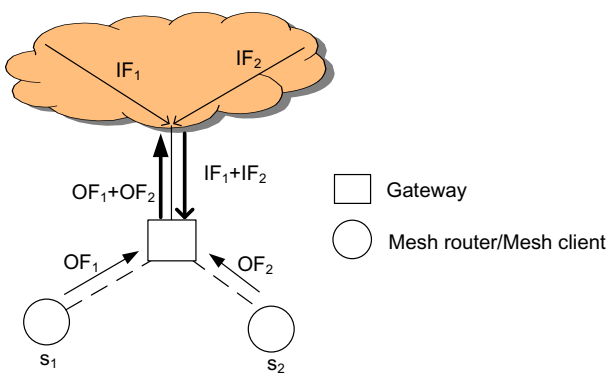

Fig. 2. An example of a busy Internet access gateway.

link $\left(I_{l}\right)$. Low $I_{l}$ is achieved by minimizing the interference and contention that results from overlapping redundant multicast transmissions by neighboring nodes. Fig. 3 illustrates an example situation where redundant transmissions between sibling nodes can interfere with each other's reception. $A$ and $B$ are suffering from interference (shown by the red flash in the figure) with the simultaneous desired downstream transmission from parent node E. Redundant transmissions can also cause interference at successive nodes. In Fig. 3, the desired downstream transmission from $A$ to $C$ competes and interferes with the redundant upstream transmission from $C$ to $A$, and the desired downstream transmission from $B$ to $D$ competes with the redundant upstream transmission from $D$ to $B$. Hence, the third objective is to decrease $I_{l}$ caused by interference and contention in these two situations. In the following section, we present our contributions in the form of network algorithms that satisfy these stated objectives.

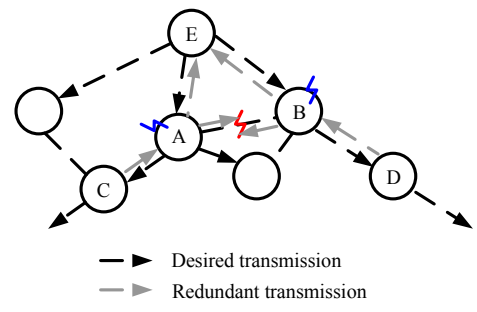

Fig. 3. An example of interference when multicasting video streams.

\section{Resource-aware Multi-Gateway WMN Video Multicasting}

In this section, we present the resource-aware multi-gateway WMN video multicasting scheme that combines a hierarchical architecture with a set of load-aware routing protocols to improve the overall video traffic capacity in large-scale WMNs.

\section{A. Overview}

We first give an overview to the multicast architecture and the multicast routing constructed by the resource-aware multigateway WMN video multicasting scheme. 
- Two-tier integrated architecture (TIA): The two-tier integrated architecture exploits wired resources through constructing access areas that join a wired overlay multicast in the upper tier as individual units. Fig. 4 illustrates an example of the hierarchical architecture. In contrast to the mini-groups in $[10,11]$ that incorporate mesh nodes which share the same closest gateway, the construction of access areas places lower demand on wired resources by maximizing the use of wireless communication where appropriate.

- Weighted gateway uploading (WGU): Unlike prior work[10,11], each access area in our scheme comprises multiple gateways. The weighted gateway uploading algorithm executed by a video source uses a combination of hop distance and load levels to avoid a nearby "busy" gateway and dynamically choose a slightly farther "nonbusy" gateway (for inter-access area routing)among the set of gateways belonging to its access area.

- Link-controlled routing tree (LCRT): LCRT routes traffic within each access area so as to reduce interference and channel contention. To achieve this objective, the LCRT algorithm computes the least number of forwarding nodes that can cover all immediate downstream nodes to form a distribution tree in each access area.

- Alternative channel assignment (ACA): The alternative channel assignment assigns channels to the two radios (sending and receiving) on each mesh node. It alternates the channel assignment at successive nodes to reduce the interference and contention caused by the transmission of a video stream.

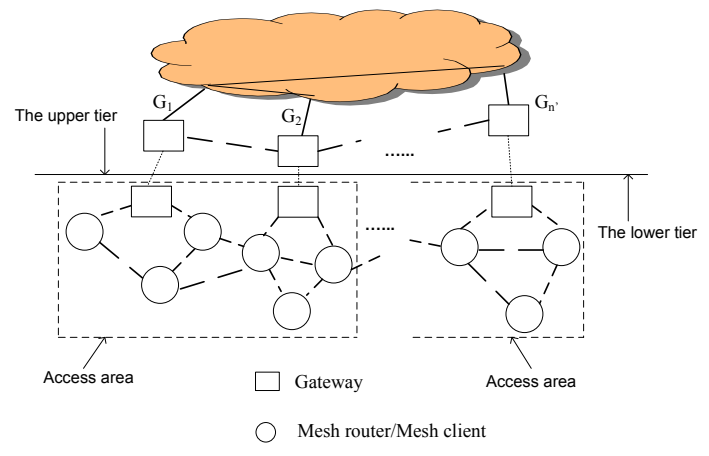

Fig. 4. An example of the two-tier integrated architecture.

\section{B. Two-Tier Integrated Architecture}

As we have introduced in the Introduction (Fig. 1), considering the already existed Internet traffic burden, the reasonable and efficient utilization of Internet shortcuts is necessary. Distant users will likely benefit from employing an Internet shortcut but close users will likely prefer intra-mesh routing. For a group of multicasting members who distribute in large-scale areas, the path selection should consider the preference of individual members with different distances to the senders. The two-tier integrated architecture is designed to facilitate this selection of paths. As illustrated in Fig. 4, all of the group members join in the lower tier where they are separated into access areas. In a given access area there is one area gateway that is selected from the gateways covered by that area. Area gateways form the upper tier in which group members belonging to the same access areas share the same wired links to communicate with group members in other areas.

1) Access Area Construction: The access area construction algorithm clusters WMN nodes into individual access areas, such that the diameter (hop distance between nodes within the access area) of each access area does not exceed a maximum permitted threshold, $K$. In practical systems, $K$ is determined by the choice of radio interfaces/channels, the propagation environment, the video data rate, and the QoS desired by the application.

The first access area is constructed by the source (denoted as $s$ ) of the video stream $V$. The protocol works as follows. $s$ broadcasts a AREA_CONSTRUCTION request packet that includes three fields: the area id of the constructing access area, the address of the packet sender/forwarder, and the packet's $T T L$. $T T L$ is initially set to $K$ by $s$. Group members who receive AREA_CONSTRUCTION set their area ids as the same one in the request packet, and then check the value of $T T L$. If $T T L>1$, they update $T T L=T T L-1$ in AREA_CONSTRUCTION and rebroadcast the packet, thereby flooding over the $K-$ hop neighborhood. At the same time, these members send a JOIN_REPORT packet to $s$, including its node type (i.e., gateways or mesh nodes), address and hop distance to $s$. The feedback allows $s$ to learn about the newly joined members.

2) Other Gateway-Initiated Access Areas: After the first access area (i.e., the source access area) is constructed, $s$ uses the weighted gateway uploading algorithm to select an uploading gateway from all its candidate gateways. This gateway is responsible for selecting area gateways before it multicasts $V$ to the gateways in the group through wired connections. An area gateway acts as a virtual source injecting traffic into the mesh, and thus constructs its own access area (similar to that constructe by $s$ ).

To select area gateways, the uploading gateway runs a protocol that broadcasts SEARCH packets that include the IP address of the packet sender, $V$ 's group id and the revised threshold of the number of wireless hops which is $(K-k)$, where $k$ is the number of wireless hops from $s$ to its uploading gateway; this ensures that the number of wireless hops is always restricted to $K$. The destinations who have $V$ 's group id but have not joined in any access area acknowledge their states to the SEARCH sender (the uploading gateway). After a period (say $T$ ), the SEARCH sender stops waiting for acknowledgement and selects the closest acknowledged gateway as a new area gateway. This new area gateway implements 
two procedures successively: a) using the algorithm that $s$ does to construct a new access area with $(K-k)$ as the threshold of the number of wireless hops, and b) searching another area gateway by using the same way that the uploading gateway does. At each time when a new area gateway is found, it feedbacks SEARCH_ACK to the uploading gateway along the reverse path from which it receives SEARCH. SEARCH_ACK includes the states of the gateways covered by the area gateway's access area. The above procedure continues until all of the group members join in an access area. The construction of the upper tier is completed when the uploading gateway knows about all of the group gateways.

Multicasting in the upper tier uses an overlay multicast technique (e.g., NICE in [17] and DSCT in [18]) to deliver $V$ from the uploading gateway to all other group gateways. The multiple gateways provide the flexibility for dynamic group management. When a new member wants to join in $V$ 's multicast, it reports to its closest gateway. After the closest gateway recognizes the existence of the new member, the new member can receive data directly from this closest gateway.

3) Dynamic Access Area: We use the threshold of the number of wireless hops $(K)$ to decide the range of an access area. The value of $K$ is initially decided based on the history of transmission in the WMN system. During multicasting $V, K$ is dynamically updated based on the performance received by group members. If a member cannot achieve an acceptable QoS level for its video, it sends QUALITY_REPORT to the report destination, which is the sender $s$ if the member is in the source access area or the area gateway if the member is in a non-source access area. QUALITY_REPORT includes the IP addresses of the member and the report destination, and the number of wireless hops from the member to the report destination.

After $s$ or an area gateway receives QUALITY_REPORT, it changes the threshold of the number of wireless hops $K$ to guarantee the quality acceptable intra-mesh video transmission within access areas. There are two cases:

Case1: If the destination of QUALITY_REPORT is $s$, the unacceptable quality report affects the communication of all access areas. $s$ first updates $K=h-1$, where $h$ is the number of wireless hops from $s$ to the report sender. It means that video packets sent by $s$ will change their TTL into $h-1$ to limit the intra-mesh routing to an acceptable range. Then, $s$ sends the updated $K$ to its uploading gateway who then multicasts the change to each area gateway. Area gateways update their threshold of the number of wireless hops as $h-$ $1-k$ which shows the video packet transmission range is changed to $h-1-k$ hops away from the area gateways.

Case2: If the destination of QUALITY_REPORT is an area gateway, the unacceptable quality report only affects members in the area gateway's access area. That is, the area gateway changes its threshold of the number of wireless hops as $h-1-k$ which limits the video packet intra-mesh routing in a range with $h-1-k$ hops away from the area gateway.

The process of shrinking the range of access area may leave some group members uncovered by any access area. The destination of QUALITY_REPORT sets these members' states as pending. Whenever the network conditions improve, they will admit these pending members back into their access areas.

\section{Weighted Gateway Uploading}

The weighted gateway uploading selects an uploading gateway through which a source can send its video streams to other access areas. The algorithm assigns each reachable gateway (i.e. a gateway in the sending access area) a weight and selects the gateway with the largest weight as the uploading gateway. In consideration of a desire for real-time and high-throughput uploading, the weight is a function of the gateways' available capacity and their distance from the video source.

To obtain the available capacity of each reachable gateway, $s$ operates a protocol which broadcasts a gateway selection request GATEWAY_SEARCH within its access area. Each gateway who receives $s$ 's request replies with a CAPACITY_REPORT with three fields: the IP address of the gateway, the available capacity of the gateway's wireless link, and the distance of the gateway to $s$. The available capacity is assessed by each gateway based on the transmission rate that the gateway currently provides. After a period (say $T^{\prime}$ ), $s$ calculates the weights for the $G^{\prime}$ gateways that replied to its request by using the following equation:

$$
w_{i}=\frac{C_{i}}{h_{i}} \text { for } i \in\left[0, G^{\prime}-1\right],
$$

where $C_{i}\left(C_{j}\right)$ is the current available capacity of the $i$ th ( $j$ th) gateway, and $h_{i}\left(h_{j}\right)$ is the distance (represented by the number of wireless hops) of the $i$ th ( $j$ th) gateway to $s$.

Equation (2) shows that the selection of uploading gateway uses a load-distance balanced metric to find a "non-busy" and close gateway. This approch helps the sender to route its video traffic around 'bottleneck' gateway nodes that might be overloaded handling traffic for other flows. It shows that the weighted gateway uploading algorithm is able to deliver video traffic between access areas with low $\delta_{T}$ and $\delta_{J}$.

\section{Link-controlled Routing Tree}

The link-controlled routing tree algorithm is run by the source in the sending access area or by the area gateways in other access areas to construct a routing tree that multicasts packets in that access area. The motivation of the algorithm is to decrease interference by selecting the least number of forwarding nodes that can connect all of the multicast receivers in the access area. Before describing the algorithm, we define the following terms.

- Node level is defined as the least number of wireless hops from the node to its closest gateway. A $l$-level node has at least $l$ wireless hops to its closest gateway. As 
an example, in Fig. 5, $A, B, C$ and $D$ are 1-level nodes because they only need one hop to their closest gateways, and $E, F$ and $G$ are 2-level nodes because of their 2 hops to the closest gateways.

- A node's uncovered out degree refers to the node's number of direct child members who have not found their forwarders. For example, in Fig. 5, the uncovered out degree of $G_{2}$ is 0 .

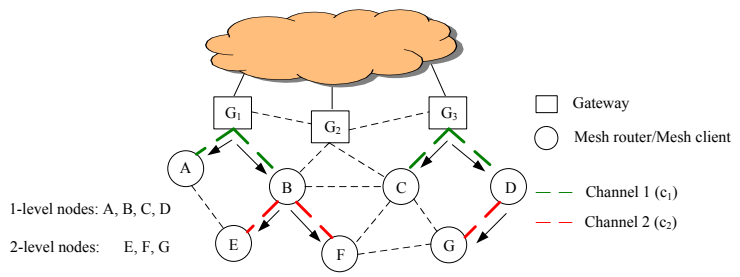

Fig. 5. An example of the link-controlled routing tree.

The link-controlled routing tree is constructed based on each node's uncovered out degree and available output capacity. The tree in the sending access area has one root (i.e., the source $s$ ), but in the non-sending access areas has multiple roots (i.e., more than one gateway covered by the access area). As we have introduced, during the access area construction, the source $s$ of the area gateway obtains the information of the members covered by its access area, including the node level for each node. The tree construction begins at the members who have the largest levels (say $L$ ). All of the $L$-level members are on-tree leaf members. To search the forwarders for these leaf members, the algorithm checks all of the $(L-1)$ level members. At each time, a $(L-1)$-level non-forwarder member who 1) has the largest uncovered out degree and the available capacity to output $V$ and 2) is not a neighbor of any selected $(L-1)$-level forwarder has priority to be selected as a forwarder. When the selected $(L-1)$-level forwarders cover all $L$-level members, the algorithm stops searching for additional $(L-1)$-level forwarding nodes. If the $(L-1)$-level forwarders selected based on the two conditions cannot cover all $L$-level members, the algorithm will select more forwarders only based on the first condition to guarantee that each $L$-level member has a forwarder.

For example, in Fig. 5. nodes $E, F$, and $G$ are leaf nodes because they have the largest level values. Their forwarders will be selected from the 1-level members. Among the 1-level members, $B$ and $C$ has the same uncovered out degrees which are all 2. A node with larger available output capacity ( $B$ in this example) will be selected as a forwarder. Then, $D$ is selected as another forwarder by the algorithm because $C$ is $B$ 's neighbor. When the selected forwarders connect all of the members in their immediate lower level, the algorithm goes to search forwarders for the $(L-1)$-level forwarders among all $(L-2)$-level members. In Fig. 5, $G_{1}$ and $G_{3}$ are selected as forwarders. When each area member has its on-tree forwarder, the link-controlled routing tree is constructed.

Algorithm 1 Link-Controlled Routing Tree
Input: An access area, the source $s$ in the sending access
area, or the area gateway $A G$ in a non-sending access area.
Output: The link-controlled routing tree in the access area; 1. s/AG obtains each area member's address information and its hop distance to $s / A G$ during the access area construction; 2. s/AG assigns a node level to each member according to its hop distance;

3. $s / A G$ sets the members that have the highest levels as leaf nodes, and $l=L-1$; // $L$ is the value of the highest level 4. While $l>0$

5. While $u m_{l} \neq 0 / / u m_{l}$ is the number of uncovered members in the $(l+1)$ th level

6. $s / A G$ searches a $l$-level member who has a) the largest uncovered out degree and b) the least connections to the selected $l$-level forwarders, and then informs the $l$-level member to become a forwarder;

7. $s / A G$ removes $m$ members who are in the $(l+1)$ th level and covered by the selected forwarder from the uncovered member set, and updates $u m_{l}=u m_{l}-m$;

8. $\quad l=l-1$;

By choosing the nodes with the largest uncovered out degree as forwarders, the link-controlled routing tree algorithm exploits wireless broadcast advantage to reduce the resource usage of the network. Meanwhile, the neighborhood of sibling forwarders is greatly avoided and therefore the interference/conflict when multicasting video in parallel. It is good for real-time (i.e., low $\delta_{D}$ ) wireless video multicasting.

\section{E. Alternative Channel Assignment}

The goal of the alternative channel assignment algorithm is to guarantee video streaming transmission at two successive nodes. The algorithm utilizes the multi-channel multi-radio advantage of WMN to achieve this goal.

Each group member of the video multicasting from $s$ are assigned two different radio interfaces that are set as the sending and receiving channels alternatively at successive nodes. Channel assignment begins at the source $s$ in the sending access area and the area gateway $A G$ in non-sending access area. s/ $A G$ chooses one channel (say $c_{1}$ ) at random as its sending channel, and the other $\left(c_{2}\right)$ as its receiving channel. Then, $s / A G$ broadcasts its channel decision through the sending channel $c_{1}$. Meanwhile all other nodes are listening to both channels. The nodes which pick up $s / A G$ 's channel decision then choose their own channels so as to be complementary. In this case, they will choose $c_{1}$ to receive and $c_{2}$ to send. The nodes to which they transmit will in turn receive on $c_{2}$ and send on $c_{1}$. In this fashion, the choice of sending and receiving channels will alternate, switching roles at each level 
of the routing tree. For example, in Fig. 5, if $G_{1}$ and $G_{3}$ pick up $c_{1}$ as the sending channel and $c_{2}$ as the receiving channel, their direct child members $B$ and $D$ will use $c_{1}$ to receive and $c_{2}$ to send. In this way, $G_{1}, G_{3}, B$, and $D$ can forward video streams continuously.

The alternative channel assignment algorithm greatly decreases interference caused by the streaming transmission at successive nodes. One reason that we utilize two different radio interfaces instead of more numbers of different radio interfaces is because of the simple assignment control which generates low control overhead. Furthermore, the linkcontrolled routing tree greatly decreases the probability that neighboring members belong to the same level. It is very rare on our routing tree that a member connects to more than one upstream forwarders. Hence, the use of greater numbers of channels would not be expected to yield significant additional benefit. In multiple radio interface WMN systems, other radio interfaces that $s$ is not using are reserved for video multicasting from other sources. When group members of different sources are not overlapping, the same radio interfaces can be used by different video multicasting without capacity cost. When group members of different sources are densely overlapping, video multicasting from different sources can employ different radio interfaces. Further, in our next step, we are interested in studying that different video multicasting coming from the same access area can use the same channel assignment through scheduling since their group members are densely overlapping.

\section{Performance Evaluation}

This section presents results on using the discrete event simulator ns2 [19] to study the performance video multicasting in WMNs. We studied the following four multicast schemes in our simulation:

1) EM: The integrated multicasting algorithm proposed in [8]

2) IR: Intra-mesh routing using wireless media broadcasting to deliver packets

3) RMG: Our proposed integrated multicasting algorithm which include TIA, WGU, LCRT, and ACA (see Section IV)

4) IW: A reduced version of our integrated multicasting algorithm which includes only TIA and WGU

We evaluate the performance of the above multicasting schemes using the following metrics:

- Average multicast delay (AMD). AMD is used to evaluate the real time of video multicasting. In our simulation, it is calculated by

$$
A M D=\frac{\sum_{i=0}^{n-1} A D_{i}}{n},
$$

where $A D_{i}$ is the average packet delay at the $i$ th member, and $n$ is the group size.

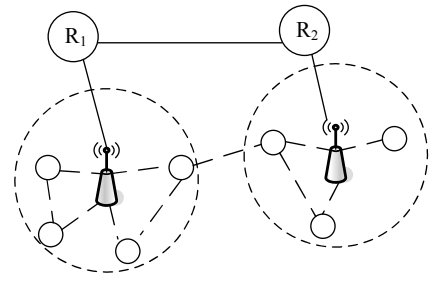

Fig. 6. The topology of small-size WMN

- Average multicast throughput (AMT). AMT is used to evaluate the quality of the multicating video streams. In our simulation, it is calculated by

$$
A M T=\frac{\sum_{i=0}^{n-1} A T_{i}}{n},
$$

where $A T_{i}$ is the average packet throughput at the $i$ th member.

- Average multicast delay jitter (AMDJ). AMDJ is observed to evaluate the video streams' continuous transmission during multicasting. Smaller AMDJ is expected. In the simulation, AMDJ is calculated by

$$
A M D J=\frac{\sum_{i=0}^{n-1} A D J_{i}}{n},
$$

where $A D J_{i}$ is the average video delay jitter at the $i$ th member.

In all simulation, the wireless links use 802.11 as defined in $\mathrm{ns} 2$ and the rate of the packetised video is $128 \mathrm{kbit} / \mathrm{s}$. Each simulation result is the average of 10 simulation runs which last for 80 seconds each.

\section{A. Evaluation using a small-scale WMN}

In this section, we study the performance of the various multicast schemes using the small-scale WMN shown in Fig. 6 where the wired and wireless links are shown with, respectively, solid lines and dashed lines. The integrated network consists of two routers $\left(R_{1}\right.$ and $\left.R_{2}\right)$ connected by a wired link with a bandwidth of $1000 \mathrm{Mbit} / \mathrm{s}$. The WMN gateways connect to the routers using a wired link with bandwidth 1000Mbit/s. For RMG scheme, each WMN node has 2 single-channel radio interfaces in RMG, while each each node has 1 radio interface in EM, IR and IW.

1) Impact of multicast group size on performance: In this experiment, we vary the size of the multicast group from 18 to 44 members to study the impact of this on the network performance in terms of average multicast delay, average multicast throughput, and average delay jitter. The results shown here are the average of 10 runs.

Fig. 7 shows the average multicast delay of the four multicast schemes when the multicast group size changes. Note that both RMG and IW have longer average multicast delays than 


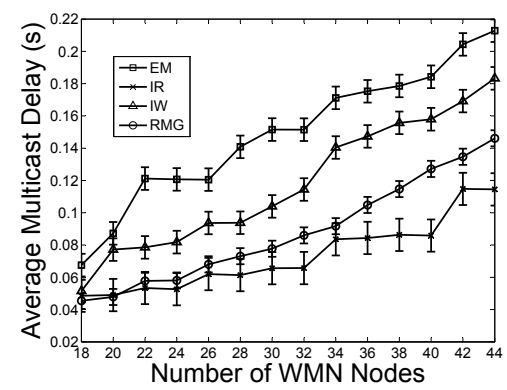

Fig. 7. The average delay performance in the small-size WMN of Fig. 6.

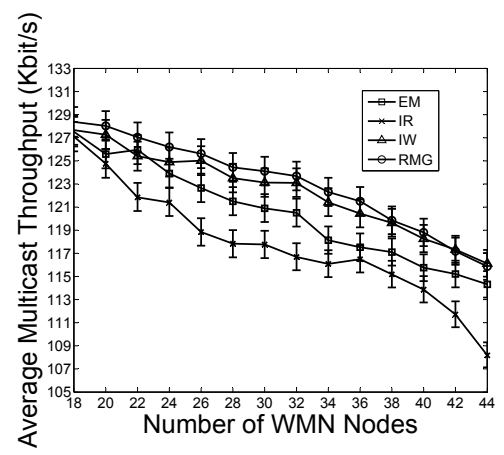

Fig. 8. The average throughput performance in the small-size WMN of Fig. 6.

IR because their overhead to construct a multicast tree. The reduced delay of RMG, in comparison with IW, shows the the positive effect of LCRT and ACA algorithms in efficiently sharing wireless resources. Note that EM gives the longest delay because it does not use wired resources in an efficient way. These results prove wired resources should be used judiciously in order to improve performance.

Fig. 8 shows the average multicast throughput of the four multicast schemes. It shows that the integrated schemes (EM, IW and RMG) achieve a higher throughput than IR because they utilise the stable and high-capacity wired connection. Note that RMG achieves a higher throughput than IW due to its use of LCRT and ACA. Note also that both RMG and IW perform better than EM because of the use of the weighted gateway uploading algorithm.

Fig. 8 shows the average delay jitter for the four schemes. IR has the worst performance while RMG has the best performance. The large performance gap between RMG and IW can be explained via the use of the link-controlled routing tree algorithm in RMG to maintain the continuous flow of the video streams.

\section{B. Evaluation using a large-scale WMN}

This section presents simulation results to evaluate the performance of various multicasting algorithm using a largescale WMN topology consisting of both wired and wireless

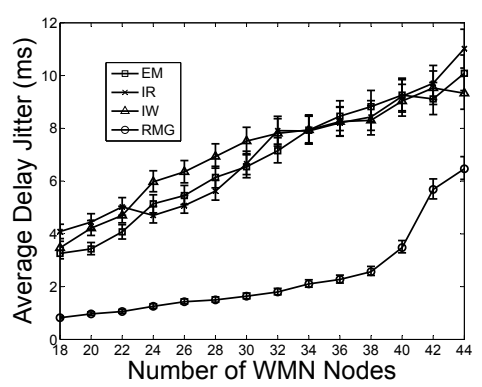

Fig. 9. The average delay jitter performance in the small-size WMN of Fig. 6.

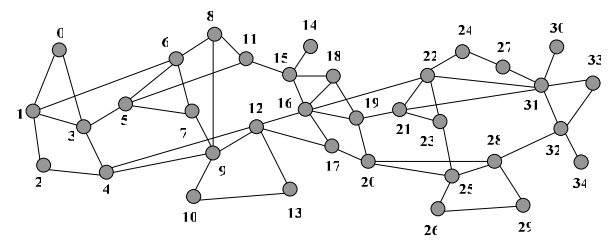

Fig. 10. The wired backbone in the simulation.

networks. The wired part of the integrated WMN topology is obtained from merging two MCI-ISP backbone networks and is shown in Fig. 10, A router in the topology represents a domain and connects to other routers through 1000Mbit/s wired links. There are totally 35 inter-domains in the wired network. The wireless part is a WMN consisting of gateways and mesh nodes. The WMN gateways are connected to the wired router through wired link with capacity $1000 \mathrm{Mbit} / \mathrm{s}$ and are connected to mesh nodes via wireless connections. We assume in the simulation that each WMN node using RMG has 2 single-channel radio interfaces, while each node in EM and IW only has 1 radio interface. Note that we did not use IR in the simulation since it is not practical to use IR in such as large-scale network.

1) Impact of multicast group size on performance: This section studies the impact of the size of multicast group on the performance of the four multicast schemes. In the simulation, the size of the multicast group increases from 130 members to 310 members with the members spread across 15 domains in Fig. 10. The average multicast delay of the three multicast schemes are shown in Fig. 11. Note that RMG achieves a much smaller delay than EM and IW for a large range of multicast group size. In addition, the average delay of EM and IW increases sharply with increasing multicast group size, while the average delay of RMG remains small. This is because RMG minimises interference and contention by enabling simultaneous video transmission at neighboring nodes using ACA.

Fig. 12 shows the average multicast throughput for EM, IW, and RMG when the multicast group size increases from 130 to 310 members. The throughput for EM drops quickly 


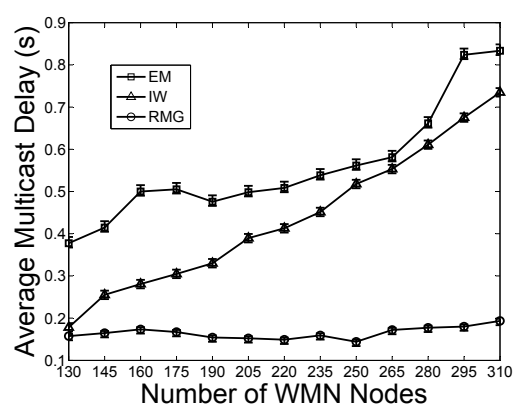

Fig. 11. The average delays when the group size increases.

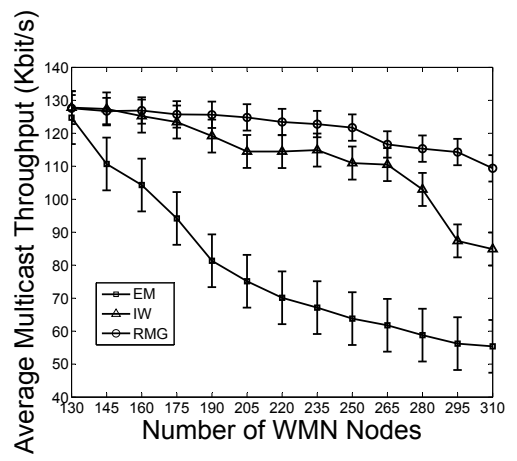

Fig. 12. The average throughput performance when the group size increases from 130 to 310 .

with increasing multicast group size because it does not use multiple gateways to distribute videos. The busy gateways soon become bottleneck of video multicast. By using weighted gateway uploading, IW gives at most $73.1 \%$ more throughput than EM. Finally, with the use of both link-controlled routing tree and the alternative channel assignment, RMG performs best and its throughput is within $10 \%$ of the source video rate for all the multicast group sizes considered.

Figure 13 compares the average delay jitter of the three multicast schemes. The jitter of all three schemes increases with increasing multicast group size. However, the jitter for RMG is limited to $4 \mathrm{~ms}$. The level of jitter achieved of both EM and IW is similar and increases with the size of multicast group. For the 310-member case, RMG reduces the jitter of EM or IW schemes by about $77.8 \%$. These results prove the advantages of using the alternative channel assignment and the link-controlled routing tree in RMG since they can greatly reduce the interference by exploiting parallel transmissions. In general, the results in this section show that quality video multicasting is realised by EM, IW and RMG if the number of multicast members is no more than 140, 220 and 310 respectively.

2) Impact of number of gateways and number of nodes in an access area: This section studies the impact of the number of gateways and the number of nodes in the source access area

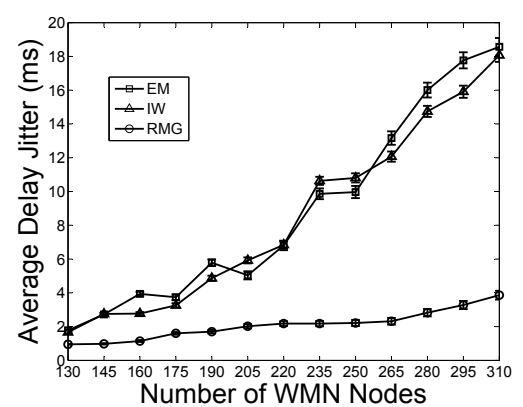

Fig. 13. The average delay jitter performance when the group size increases from 130 to 310 .

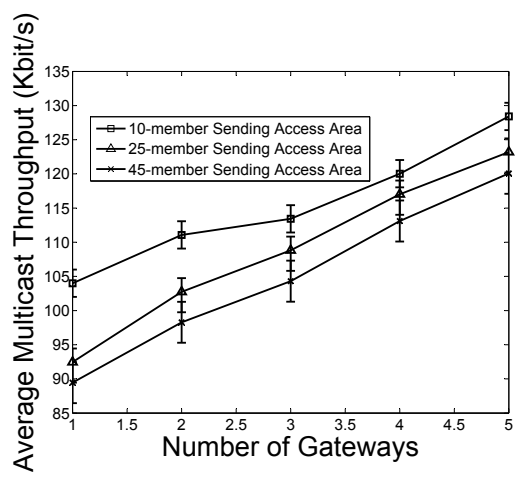

Fig. 14. The average multicast throughput performance varies with the number of gateways in the sending access area.

on the performance of RMG. We vary the number of gateways per source access area from 1 to 5 and the number of nodes in a source access area from 5 to 45 . The total number of group members in other access areas are 240.

Fig. 14 shows the average multicast throughput of RMG when the number of gateways in each source access area varies from 1 to 5 and the number of nodes in each source access area is 10, 25 and 45. The figure shows that multicast throughput increases sharply with increasing number of gateways for a fixed number of nodes in an access area. It shows that multiple gateways can significantly improve the throughput of an integrated network.

Fig. 15 shows how the number of nodes in a source access area affects the average throughput of RMG. It shows that increasing the size of a source access area has a negative effect on the throughput. However, this can be corrected by increasing the number gateways per source access area.

\section{CONCLUSIONS}

The paper studied a novel and distributed resource-aware multi-gateway video multicasting scheme. We first analyzed the communication cost that wireless mesh signals experience during wireless transmission. We then presented a set of novel algorithms which work to reduce this cost by efficiently 


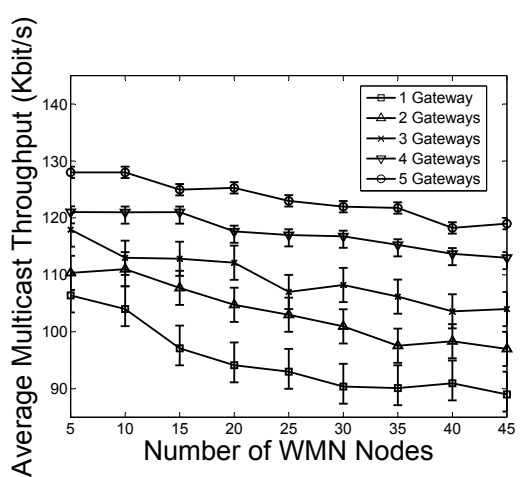

Fig. 15. The average throughput performance varies with the sending access area size and the number of gateways.

employing wired resources and reducing the possibility of quality degradation induced by wireless interference. These measures enable capacity for multicasting video to be maintained in large-scale areas. The two-tier integrated architecture algorithm chooses communication paths (intra-mesh paths or integrated paths) between group members by organizing them into a clustered and layered architecture; the weighted gateway uploading algorithm avoids "busy gateways" by uploading video in a load-distance balanced way; the link-controlled routing tree algorithm decreases interference caused by parallel multicasting by constructing a multicast tree with the least number of forwarders in each access area; and the alternative channel assignment algorithm guarantees video's streaming transmission by assigning different sending/receiving channels to successive members alternatively. Our design principles were validated by our simulation results.

In general, our scheme improves scalability of capacity in large wireless networks. It not only achieves QoS-guaranteed individual video multicasting in large scale areas but also enables more video streams to be transmitted. These improvements require the use of multiple gateways and also that the wireless distance from each source to its closest gateway does not exceed a maximum permitted threshold. The distance requirement may be guaranteed by simply placing enough gateways to connect mesh nodes, given that WMN gateways are cheap. The relative ease of deployment of modern wireless technology also aids the use of multiple gateways.

\section{ACKNOWLEDGMENTS}

This work was supported by the IRCSET Embark Initiative Postdoctoral Fellowship, and partially supported by Australian Research Council (ARC) Discovery Grant DP0664791. The work was finished when the first author was an Embark Initiative Postdoctoral Fellow in University College Cork.

\section{REFERENCES}

[1] S. Weber, X. Yang, J. Andrews, and G. Veciana. Transmission Capacity of Wireless Ad Hoc Networks With Outage Constraints. IEEE Trans. on Information Theory, Vol. 51, No. 12, December 2005.

[2] S. Shakkottai, X. Liu and R. Srikant, The multicast capacity of large multihop wireless networks. In Proc. of ACM MobiHoc '07, pp. $247-$ 255, 2007.

[3] J. Jun, and M. L. Sichitiu. The Nominal Capacity of Wireless Mesh Networks. IEEE Trans. on Wireless Communications, Vol. 10, October 2003.

[4] S. Banerjee, B. Bhattacharjee, and C. Kommareddy. Scalable Application Layer Multicast. Proc. of ACM SIGCOMM, pp. 205-217, August 19-23, 2002, Pittsburgh, Pennsylvania, USA.

[5] W. Tu, and W. Jia. A Scalable and Efficient End Host Multicast for Peerto-Peer Systems. Proc. of IEEE Globecom 2004, pp. 967-971, November 29-December 3, 2004, Dallas, Texas, USA.

[6] B. Zhang, S. Jamin, and L. Zhang, "Host multicast: a framework for delivering multicast to end users", Proc. of IEEE INFOCOM 2002, pp. 1366-1375, June 23-27, 2002, New York, USA.

[7] W. Tu, C. Sreenan, and W. Jia. Worst-Case Delay Control in MultiGroup Overlay Networks. IEEE Transaction on Parallel and Distributed Systems, Vol. 18, No. 10, October 2007.

[8] P. M. Ruiz, F. J. Galera, C. Jelger, and T. Noel. Efficient Multicast Routing in Wireless Mesh Networks Connected to Internet. in Proceedings of The First International Conference on Integrated Internet Ad Hoc and Sensor Networks, 2006, Nice, France.

[9] Y. Amir1, C. Danilov, R. M. Elefteri, and N. Rivera. An Inter-Domain Routing Protocol for Multi-Homed Wireless Mesh Networks. in Proceeding of IEEE International Symposium on a World of Wireless, Mobile and Multimedia Networks (WoWMoM 2008), 2008, Helsinki, Finland.

[10] P. M. Ruiz, A. F. Gomez-Skarmeta, and I. Groves. The MMARP Protocol for Efficient Support of Standard IP Multicast in Mobile Ad Hoc Access Networks. in Proceeding of the 1ST Mobile and Wireless Communications Summit 2003, June 2003, page 478-482, Aveiro, Portugal.

[11] P. M. Ruiz, F. J. Galera, C. Jelger, and T. Noel. Efficient Multicast Routing in Wireless Mesh Networks Connected to Internet. in Proceedings of the first international conference on Integrated internet ad hoc and sensor networks, 2006, Nice, France.

[12] Y. Amir, C. Danilov, R. Musaloiu-Elefteri, and N. Rivera. An InterDomain Routing Protocol for Multi-Homed Wireless Mesh Networks. in Proceeding of The IEEE International Symposium on a World of Wireless, Mobile, and Multimedia Networks (WoWMoM 2007), Helsinki, Finland.

[13] B. Liu, C. Chou, A. Misra, and S. Jha. Resource-aware Routing of Broadcast and Multicast in Multi-rate Wireless Mesh Networks. Mobile Networks and Applications (accepted Feb. 2008).

[14] C. Chou, A. Misra, and J. Qadir. Low Latency Broadcast in MultiRate Wireless Mesh Networks. IEEE JSAC Special Issue on Multi-hop Wireless Mesh Networks, 2006.

[15] T. Wang, X. Du, W. Cheng, Z. Yang, and W. Liu. A Fast Broadcast Tree Construction in Multi-Rate Wireless Mesh Networks. In Proc. of IEEE International Conference on Communications (ICC'07), June, 2007, Glasgow.

[16] W. Tu, and C. Sreenan. Adaptive Split Transmission for Video Streams in Wireless Mesh Networks. in Proc. of IEEE Wireless Communications and Networks Conference (WCNC 2008).

[17] S. Banerjee, B. Bhattacharjee, and C. Kommareddy. Scalable Application Layer Multicast. In Proc. of ACM SIGCOMM, page 205-217, August 19-23, 2002, Pittsburgh, Pennsylvania, USA.

[18] W. Tu, and W. Jia. A Scalable and Efficient End Host Multicast for Peerto- Peer Systems - DSCT. In Proc. of GlobeCom 2004, page 967-971, November 29 - December 3, 2004, Dallas, Texas, USA.

[19] UC Berkeley, LBL, USC/ISI, and Xerox PARC. Ns Notes and Documentation. October 20, 1999.

[20] J. Edwards, Integrated Wired and Wireless: The Best of Both Worlds, IQ Magazine, Vol. VII, No. 2, 2006 